\title{
Establishing the epidemiology of respiratory viral infections using "A NOVEL POINT-OF-CARE MULTIANALYTE ANTIGEN DETECTION TEST mariPOC" during the season 2013-2014
}

Angelica Vişann ${ }^{1,2}$, Anca Drăgănescu' ${ }^{1}$ Anuța Bilaşco ${ }^{1}$, Cristina Negulescu', Camelia Kouris ${ }^{1}$, Ruxandra Măntescu', Gheorghiță Jugulete ${ }^{1,2}$, Mădălina Merişescu ${ }^{1,2}$, Endis Osman ${ }^{1}$, Cornelia Dogaru', Diana Slavu ${ }^{\text {* }}$, Monica Luminos ${ }^{1,2}$

From The 10th Edition of the Scientific Days of the National Institute for Infectious Diseases "Prof Dr Matei Bals" Bucharest, Romania. 15-17 October 2014

\section{Background}

Rapid etiological diagnosis has a very important role in the clinical management of respiratory viral infections. Using a multianalyte point-of-care detection system, based on a fully automated immunoassay method, we can detect 8 respiratory viruses (influenza $A$ and $B$ viruses, parainfluenza 1,2 and 3 viruses, respiratory syncytial virus, human metapneumovirus and adenovirus) and the presence of Streptococcus pneumoniae from a single nasopharyngeal swab or aspirate. Objectives: To evaluate the incidence of respiratory viral infections in the pediatric department of the National Institute for Infectious Diseases "Prof. Dr. Matei Balş"during 1 November 2013 - 1 June 2014.

\section{Methods}

The collected samples from children with respiratory tract symptoms were analyzed by mariPOC (the novel multianalyte point-of-care antigen detection test). Positive samples were then studied in terms of clinical manifestations, complications, signs of bacterial co-infection, antiviral and antibiotic administration and days of hospitalization.

\section{Results}

We tested approximately 600 samples, out of which 50\% were positive for at least one virus. The most frequent infection was influenza A, which accounted for $55 \%$ of the positive samples. Other frequent viruses found were respiratory syncytial virus in $27 \%$ of cases, human metapneumovirus in $6.2 \%$. We found viral co-infections in $8.9 \%$ of cases, out of which the most frequent association was influenza A virus with respiratory syncytial virus.

\section{Conclusion}

The mariPOC antigen detection test provides a very useful and rapid pathogen specific diagnosis of respiratory infections, having a high specificity for the most important viruses. Using this method we found that influenza A virus was the most frequent viral infection in children during 2013-2014 winter-spring season, but also that viral co-infections are an important etiology of respiratory symptoms in children.

\section{Authors' details}

${ }^{1}$ National Institute for Infectious Diseases "Prof. Dr. Matei Balș", Bucharest, Romania. ${ }^{2}$ Carol Davila University of Medicine and Pharmacy, Bucharest, Romania.

Published: 15 October 2014

doi:10.1186/1471-2334-14-S7-033

Cite this article as: Vişan et al:: Establishing the epidemiology of respiratory viral infections using "A NOVEL POINT-OF-CARE MULTIANALYTE ANTIGEN DETECTION TEST mariPOC" during the season 2013-2014. BMC Infectious Diseases 2014 14(Suppl 7):O33.

\footnotetext{
* Correspondence: diana_maria_slavu@yahoo.com

'National Institute for Infectious Diseases "Prof. Dr. Matei Balş", Bucharest,

Romania

Full list of author information is available at the end of the article
} 